\title{
Smart Home Automation using Arduino Integrated with Bluetooth and GSM
}

\author{
Akash Sood, Ronak Fofaliya, Rajesh Babu Chandran
}

\begin{abstract}
As wireless technology is progressing and the usage of smartphones is increasing, a home automation tool will be a perfect consumer product that will automate the process of controlling the switches which were previously handled manually. The primary objective of this project is to develop a cost effective tool that provides the user the flexibility to control their appliances, either Bluetooth when they are at their home or by SMS when they are outside their home, to control their appliances. Arduino board is used for computation and interpretation of the incoming signals from the Bluetooth and GSM module.
\end{abstract}

\section{INTRODUCTION}

In this progressing world of technology and automation, it is only inevitable that houses get smarter by utilizing these technologies. O ur project aims to develop a cost effective home automation system using Arduino with Bluetooth and GSM modules. A Bluetooth and a GSM module is used to create this smart home network. Both modules act as receivers of the incoming signals $(\mathrm{O} \mathrm{N} / \mathrm{O} \mathrm{FF})$ that the user sends via the mobile application or through SMS. O $n$ successful interpretation of the incoming message, action is performed by the Arduino board on the connected appliances.

As smartphone usage is increasing among all age groups, it is only a matter of time that the consumers would want to control their home appliances using them. The scope of this technology is very vast as it is still open for improvement and innovation.

We propose to introduce a GSM module in our project which would enable the user to control their appliances from any part of the world via SMS from their smartphone.

\section{AUTOMATION}

Some of the home automation systems presently in use are expensive in manufacturing and support only one mode of communication. These systems have a limited range of use and lack alternative communication methods that could be cost effective as well as enable the user to access their devices from locations other than their homes.

[1] Home Automation Architecture based on IoT Technologies- Judson Costa, Daniel Araujo, Diego R. C. Silva, Marcelo B. Nogueira and Marconi C. Rodrigues. -

Research- In the extent of remote correspondence, a few prospects were created for sending data between electronic gadgets with the goal to expand safety and signal range. This

Revised Manuscript Received on September 22, 2019.

Akash Sood, SRM Institute of science and Technology

Ronak Fofaliya, SRM Institute of science and Technology

Rajesh Babu Chandran, SRM Institute of science and Technology architecture adopts: W i- Fi protocol - since it is a wireless communication network it has numerous advantages such as mobility, compatibility, sharing, accessibility, low maintenance cost, security, and popularity. Limitation- In this paper, the only medium to communicate with the smart home device is through a W ireless Fidelity network thus, limiting the usage of the device to a very short range.

[2] Smart Home Automation System Using Bluetooth Technology- Muhammad Asadullah, IEEE Student Member, Khalil Ullah, IEEE Member X Research- A low cost and user friendly remote controlled smart home system is implemented using Arduino UNO board, Bluetooth module, ultrasonic sensor and moisture sensor. A smartphone application is developed to use the devices connected to the relay. Most of conventional smart home systems nowadays are aimed for different purposes while the system proposed in this paper is used for general purposes. The proposed system has additional features than a conventional home automation system such as the ultrasonic sensor which is used for water level detection in devices such as water purifiers. Limitations- In this system, the user can only utilize the functionality of the device with Bluetooth connectivity, thus, limiting the range of usage as well as necessarily using devices that have Bluetooth modules for communication

[3] A Smart Home Automation via Facebook Chatbot and Raspberry Pi- Tussanai Parthornratt, Pasd Putthapipat, Dollachart Kitsawat, Prapap Koronjaruwat

- Department of Electrical Engineering, Department of Computer Engineering, Department of Computer Engineering, Department of Computer Engineering, Assumption University Bangkok, Thailand Research- This paper presents an untried implementation of smart home automation using Raspberry Pi, Facebook Chabot and Google Maps API. The development process was reduced and extra operations like estimated time of arrival using Google Maps, user friendly communication using Facebook Chabot and secure communications were integrated. Essentially, this home automation system is extendable and accessible without requiring an architectural remodeling and other technical hitches. The design of smart home automation system in this project is intended for simplicity and user- friendly nature. Any ordinary user would prefer to use a software that he/she is familiar with and send simple commands that deliver desired outcomes. mitations- The entire project sits on the Raspberry Pi module which makes the entire system expensive. Also, the project uses Facebook UI which requires an account for operation which may not be secure and feasible for operation. Noticing these drawbacks of shortened range, expensive computation 
boards and user complexity, we intend to develop a smart home automation device that uses a GSM Module that enables the user to operate devices while they are on the move.

\section{PROPOSED SYSTEM}

Arduino board is used to interpret the incoming signal from the Bluetooth or GSM module, after which it points to the connected device and changes it state to either $\mathrm{O} \mathrm{N}$ or $\mathrm{O}$ FF.

The Arduino board is connected to a PCB block that increase connectivity using additional on- board relays. The Bluetooth and the GSM module are connected to this PCB block using jump wires.

The entire system requires a 12 Volt wall power which does not consume excess electricity and provides reliability against surges.

An android application is installed on the smartphone of the user. It consists of toggles that have certain values assigned with them with respect to the device as well as its current state.

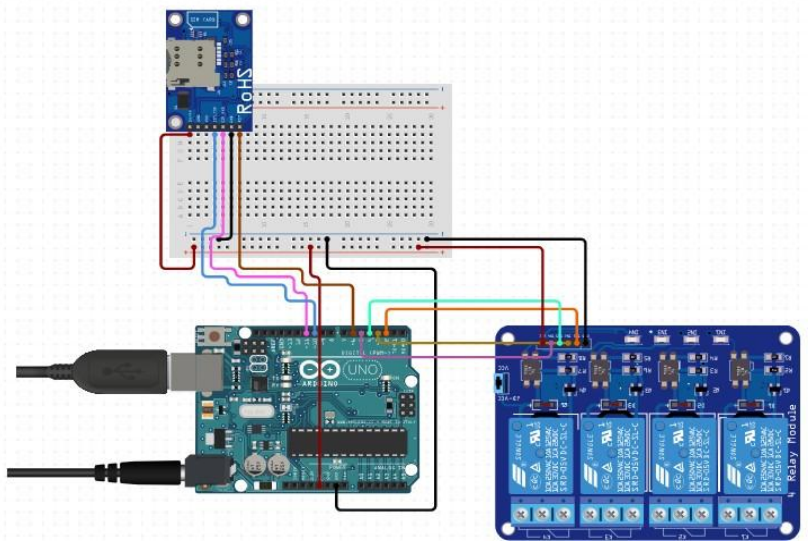

Figure 1- Proposed System Architecture

\section{SYSTEM ARCITECTURE:}

[1] Arduino Uno- MEGA 2560 is designed for complex projects. W ith 54 digital I/O pins, 16 analog inputs and it has 54 digital input/output pins (of which 15 can be used as PW M outputs), 16 analog inputs, 4 UARTs (hardware serial ports), a $16 \mathrm{MHz}$ crystal oscillator, a USB connection, a power jack, an ICSP header, and a reset button. Arduino is a cost effective alternate to other boards like Raspberry Pi. W ith sufficient power supply it can successfully operate powerful devices such as 3D printers.

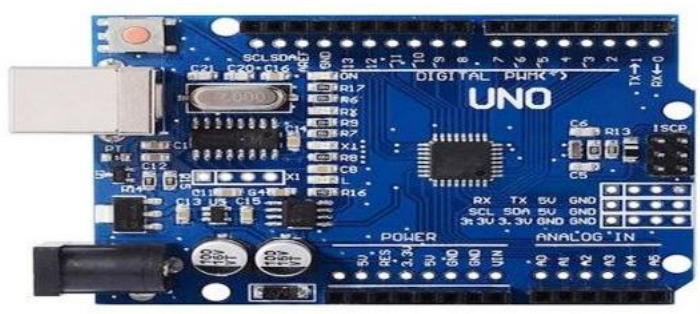

Figure 2- Arduino Uno Board

[2] Q uadBandGPRS- GSM module- SIM800L is a small quad- band GSM / GPRS module, equipped with quad- band antenna. Insert a valid SIM card and you can use it for voice calls, sending text messages and accessing the internet.

$\mathrm{HC} 1$

BLUETOOTH HC-06

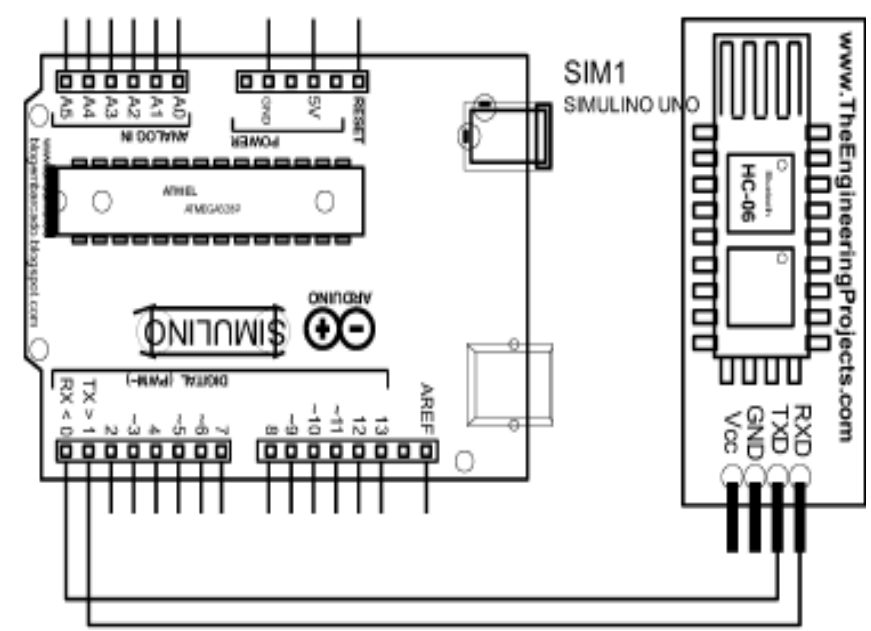

Figure 3- GSM Module Circuit Diagram

[3] W all Power- $12 \mathrm{~V}$ DC $2 \mathrm{~A}-\mathrm{A} 12 \mathrm{~V}$ power source you plug into your regular socket. W orks with 100- 240VAC inputs. The most reliable and most likely to work for all components you choose. Use it when you don' $t$ need your circuit to be mobile, or positioned in a location without wall power.

[4] Relay Module 4- Channel- 4 channel Relay module with breakout board. It is used to isolate components and create a sub- circuit in your circuit, with a different voltage.

\section{WORKING PROCESS}

The user connects to the Home network and controls the connected appliances using the application installed on his smartphone. The toggles have values assigned with respect to the appliances and their current state.

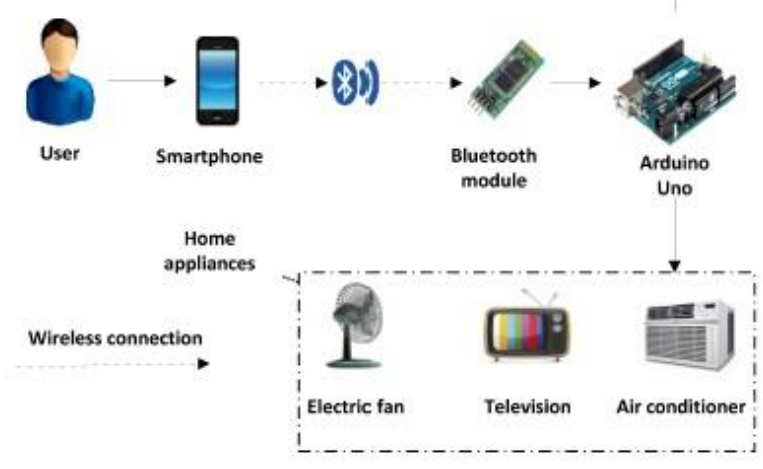

Figure 4- Working Process of Proposed System 
The Bluetooth module uses voice commands to operate the connected appliances. W hen the user says he device name with the suffix ' on' then the state of the device is changed to HIGH and vice versa for suffix ' off' .The GSM module uses a SIM Card to receive incoming messages which are then interpreted by the Arduino board and necessary action on the appliance is performed.

The flow chart for the working progress is shown below.

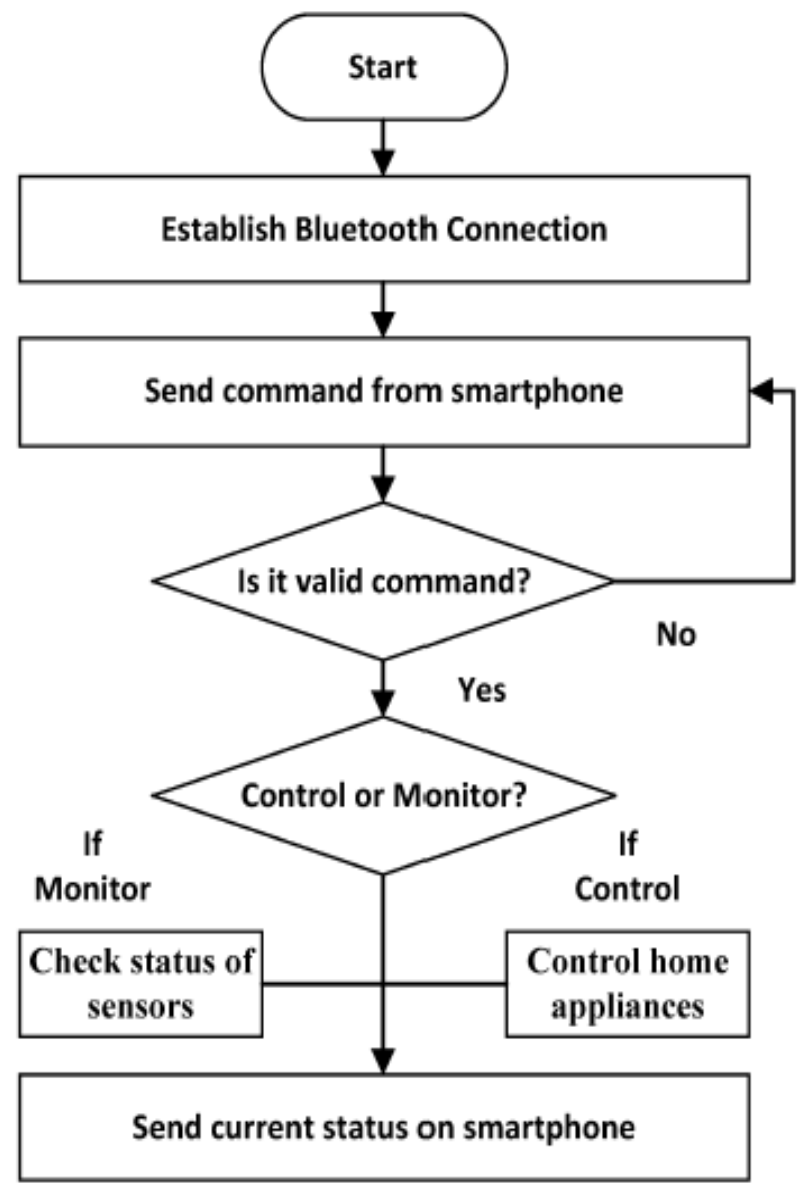

Figure 5- Flow Chart

User sends the message to the registered number of the SIM Card which is connected with the GSM module in the Arduino board.

\section{RESULT}

Using the Arduino IDE, the Bluetooth and GSM modules connected to the Arduino UNO board are configured. After initial configuration, the Bluetooth module is configured to accept the input that is sent from the Bluetooth application.

The Bluetooth module uses an application that accepts voice as its input rather than the conventional toggle buttons.

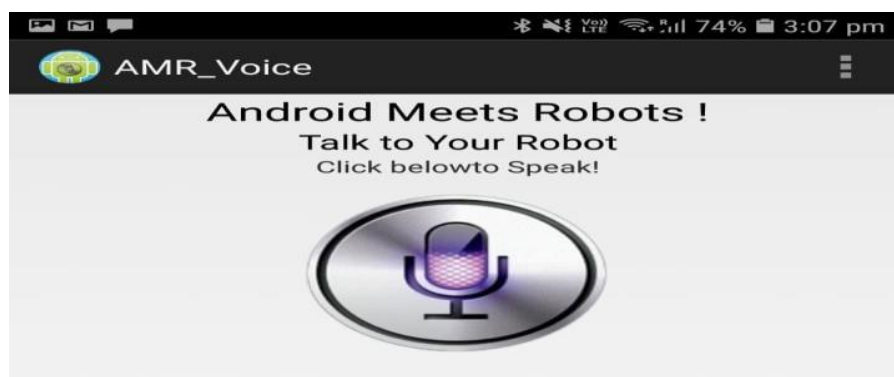

Figure 6- Bluetooth Application User Interface

[1] Create a string object to store the read input. If not null, then search for keyword $\mathrm{O}$ N/O FF in the string input by using case in sensitive search and assign $\mathrm{HIGH} / \mathrm{LO} \mathrm{W}$ value to the buzzer accordingly

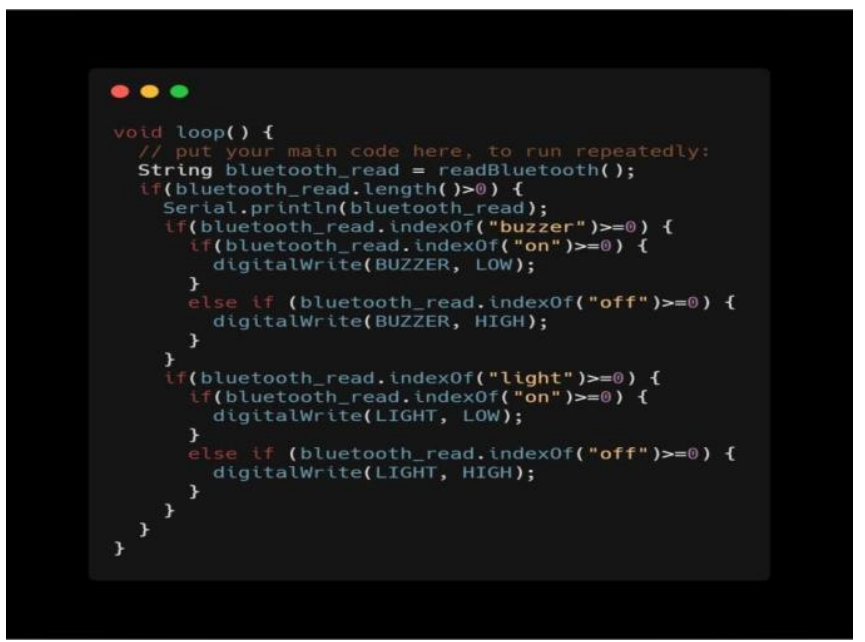

Figure 7- Code Logic For Bluetooth Module

After configuring the Bluetooth module, the GSM module is configured. It requires a SIM card and a unique remote number for its operation.

[2] After establishing the connection, sms.beginSMS() is used to send the remoteNumber of the GSM SIM. Function sms.print() is used to send the message and function sms.endSMS() is used to finish the process.

Perform operation on device using case insensitive comparison. 


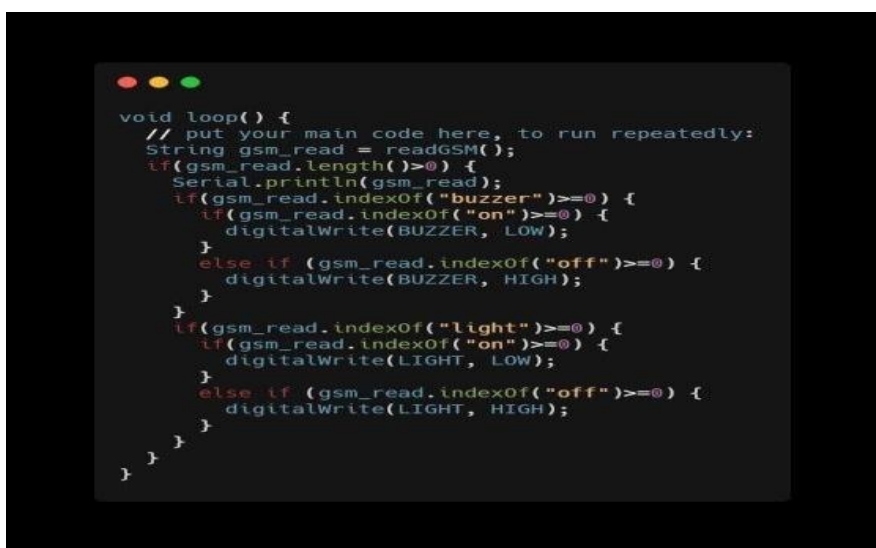

[3] Using the unique SIM number the users sends a SMS to the SIM Card on the Arduino board and the action on connected device is performed.

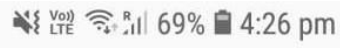

9962359915

Saturday, 2 March 2019

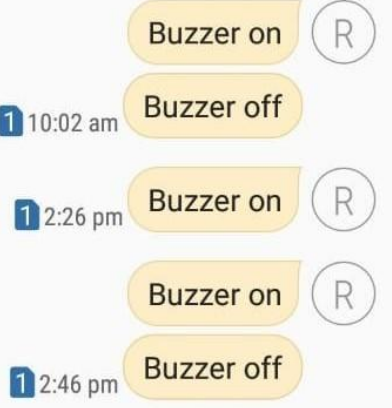

Friday, 12 April 2019

Buzzer on

$11: 12 \mathrm{am}$

Buzzer off mechanization framework for interruption location," 2015 IEEE fourteenth Canadian Workshop on data Theory (CWIT), St. John's, NL, 2015, pp. 75-78.

3. N. Skeledzija, J. C. Edin, V. Bachler, H. N. Vucemilo, H. Dzapo , "Savvy home mechanization framework for vitality proficient lodging", 37th International Convention on Information and Communication Technology, Electronics and Microelectronics (MIPRO), 2014, pp. 166-171

4. Smart Home Automation System Using Bluetooth Technology Muhammad Asadullah, IEEE Student Member(2017)IEEE Jounal.

5. M. Asadullah and A. Raza, "A review of home mechanization frameworks," 20i 6 second global Conference on Robotics and Artificial insight (fCRAi), Rawalpindi, 2016, pp. 27-31.

6. N.M.Morshed,G.M.Muid-Ur-Rahman,M.R.Karim H $\quad \mathrm{H}$ and s.U.Zaman,"Microcontrollerbasedhomeautome musingBluetooth,GSM,WiFiandDTMF,"2015International Conferenceon Advancesin Electrical Engineering(IJAEE)

7. Ashutosh Phatak ; Ruturaj Kadikar ; K. Vijayan ; B. Amutha Performance Analysis of Firewall dependent on SDN and OpenFLow-2018 IEEE Conference on Communication and Signal Processing.

8. Prathamesh Purohit; Ruturaj Kadikar ; M. Susila ; B. Amutha-Study of Service Chain Optimization in Cloud Environment.

9. B. Amutha,Swasti Saxena,Abhinaba Das-Changing Mechanisms o undertaking security-International Journal of Engineering and Technology.

10. B.Amutha,Bhawani Ghanta,Karthik Nanamaran, Manickavasagam Balasubramanian-ECOSENSE: An Energy Consumption Protocol for Wireless Sensor Networks- 2015 International Conference on Recent Trends in Copmuting

11. S.Palaniappan,N.Hariharan,N.TKesh,V.S,and A.Deborah S,"Homeautomationsystems,"InternationalJournalofCom puterApplications, vol.116,no.II,pp.11-18,Apr.2015

\section{REFERENCES}

1. K. Mandula, R. Parupalli, C. A. S. Murty, E. Magesh and R. Lunagariya, "Versatile based home mechanization utilizing Internet of Things(loT)," 2015 International Conference on Control, Instrumentation, Communication and Computational Technologies (ICcrCCT), Kumaracoil, 2015, pp. 340-343.

2. D. Chowdhry, R. Paranjape and P. Laforge, "Savvy home 